\section{Why is a paediatric respiratory specialist integral to the paediatric rheumatology clinic?}

Systemic connective tissue diseases (CTDs) are characterised by the presence of autoantibodies and multiorgan involvement. Although CTDs are rare in children, they are associated with pulmonary complications, which have a high morbidity and mortality rate. The exact pathophysiology remains unclear. The pleuropulmonary complications in CTD are diverse in their manifestations and are often complex to diagnose and manage.

The most common CTDs are discussed. These include juvenile systemic lupus erythematosus, juvenile dermatomyositis, juvenile systemic sclerosis, Sjögren's syndrome and mixed connective tissue disease. We describe the clinical features of the pleuropulmonary complications, focusing on their screening, diagnosis and monitoring. Treatment strategies are also discussed, highlighting the factors and interventions that influence the outcome of lung disease in CTD and pulmonary complications of treatment.

Early detection and prompt treatment in a multidisciplinary team setting, including respiratory and rheumatology paediatricians and radiologists, is paramount in achieving the best possible outcomes for these patients.

\section{Educational aims}

- To discuss the pulmonary manifestations in children with CTD.

- To outline the diagnostic modalities used to diagnose and monitor pulmonary manifestations in CTD.

- To review the various treatment strategies in CTD-related lung disease and the importance of a multidisciplinary approach to management.

@ERSpublications

Pleuropulmonary complications of CTD, though rare in paediatrics, can be associated with high morbidity and mortality. Joint management by respiratory and rheumatology paediatricians is recommended. Treatment includes steroids and other immunomodulators. https://bit.ly/2MCvEpp
Cite as: Ramphul M,

Gallagher K, Warrier K, et al. Why is a paediatric respiratory specialist integral to the paediatric rheumatology clinic? Breathe 2020; 16 : 200212.

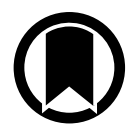

CrossMark

(C)ERS 2020 
Connective tissue diseases (CTD) encompass a group of heterogeneous systemic inflammatory diseases characterised by the presence of circulating autoantibodies and autoimmune-mediated multiorgan system involvement [1]. These diseases are rare in children [2]. Amongst the most common are juvenile systemic lupus erythematosus (JSLE), juvenile dermatomyositis (JDM), juvenile systemic sclerosis (JSSc) and mixed connective tissue disease (MCTD).

Other conditions encountered in the paediatric rheumatology service can also be associated with pulmonary manifestations, for instance systemic vasculitis and autoinflammatory disorders such as systemic juvenile idiopathic arthritis.

Clinical presentations of autoimmune CTD are extremely variable and can be associated with many systemic complications including pulmonary complications [1]. It is difficult to assess the true prevalence of pulmonary complications in CTD as they are often influenced by the acuity, chronicity and complexity of the causative CTD. The entire pulmonary system is vulnerable to injury, and interstitial lung disease (ILD) is one of the sinister complications.

Many children are initially asymptomatic from a pulmonary perspective, and unless clinicians are vigilant with a high degree of clinical suspicion, these complications may go undetected. This will enable early diagnosis and management where pulmonary involvement occurs in the context of a known diagnosis of CTD. Very occasionally, a diagnosis of CTD may come to light when a child/ young adult with diffuse lung disease is being investigated as pulmonary involvement may be the first or only manifestation of CTD.

Both respiratory and rheumatology paediatricians need a good understanding of how to monitor, investigate and manage these diseases appropriately, because early therapy may help prevent serious and permanent sequelae, thus improving patient outcomes [3]. This is best achieved with joint specialist paediatric rheumatology and respiratory reviews in a multidisciplinary setting [3]. In addition, clinicians need to be aware of pulmonary complications of the immunomodulatory treatments, including increased susceptibility to infection and development of ILD as complications of therapy.

In this review, we discuss a variety of paediatric autoimmune CTDs, highlight their clinical presentations, potential pulmonary complications, and provide an update on treatment options.

\section{Incidence of pleuropulmonary complications in CTD}

ILD is one of the most common and clinically significant pulmonary manifestations of CTD. ILD in children is a rare condition in general, with an incidence of less than one per 100000 [4], compared with 30 per 100000 in adults, of which CTD-related lung disease accounts for a small proportion.

\section{Pathophysiology}

As expected in a multisystem disease, the entire pulmonary system is vulnerable to injury. Any of its compartments including chest wall, pleura, vasculature, airways and parenchyma may be independently or simultaneously affected. The pathophysiology is not completely understood and is believed to be multifactorial [5].

There is an inflammatory immune response driven by autoantibodies [6]. The lungs are exposed to external stimuli such as microorganisms and toxins, and as the lungs are highly vascular, an inflammatory process is triggered. Macrophages act as antigen presenting cells, ingesting the inhaled antigenic stimuli and causing an inappropriate activation of autoimmune CD4 T-lymphocytes and B-lymphocytes. Several distinct immune cell populations contribute to ILD [6, 7].

There is a profibrotic process which causes irreversible remodelling of the lung [8]. Stimuli like cigarette smoke, radiation, cytokines and oxidative stress can cause epithelial-mesenchymal transition. The epithelial cells lose features such as cell-to-cell adherence, gain migratory and mesenchymal properties, and eventually convert into fibroblasts and myofibroblasts producing extracellular matrix, which engenders the profibrotic repair programme [9].

In addition to these immunological and fibrotic processes, genetic factors and microbiological triggers are also believed to contribute to the pathogenesis of lung disease in CTD, particularly ILD. These changes essentially impair lung function and potentially can result in abnormal gas exchange caused by the restructuring of the interstitial spaces of the lungs [7].

The underlying disease process influences which part of the respiratory system will be predominately affected, for example the pleura shares structures and function similar to the joint synovia, and subsequently it is often the site where inflammation happens [3]

\section{Juvenile systemic lupus erythematosus}

JSLE is an autoimmune disease, driven by autoantibodies resulting in multisystem inflammation and multiorgan involvement [10]. The incidence of JSLE is 0.3-0.9 per 100000 and it is more prevalent in non-Caucasian children [11]. It shows a female predominance after puberty, and presents in $15-20 \%$ of affected individuals before the age of 18 years [12].

JSLE is more aggressive than adult-onset disease and is linked with a higher rate of arthritis, serositis, nephritis, haematological and neurological abnormalities [13]. Within the first year of diagnosis, 
Table 1 Acute and chronic pulmonary complications of JSLE

\begin{tabular}{|c|c|c|c|}
\hline Complications & Percentage affected & Investigations & Prognosis \\
\hline \multicolumn{4}{|l|}{ Acute } \\
\hline Infective pneumonia & Up to $90 \%$ & $\begin{array}{l}\text { CXR: consolidation } \\
\text { BAL: culture may isolate pathogen }\end{array}$ & Variable \\
\hline Pleuritis & $50-80 \%$ & CXR: may show pleural effusion & Good \\
\hline Thromboembolic disease & Variable & $\begin{array}{l}\text { CXR: pulmonary oligaemia, peripheral } \\
\text { wedge-shaped consolidation, may show } \\
\text { pleural effusion } \\
\text { CTPA: may show pulmonary embolus } \\
\text { Doppler: may show venous embolus }\end{array}$ & $\begin{array}{l}\text { Variable } \\
\text { Can progress } \\
\text { to pulmonary } \\
\text { hypertension }\end{array}$ \\
\hline Alveolar haemorrhage $(\mathrm{AH})$ & $<2 \%$ & $\begin{array}{l}\text { Full blood count: drop in haemoglobin } \\
\text { CXR: patchy infiltrates at bases } \\
\text { BAL: blood stained, haemosiderin-laden } \\
\text { macrophages }\end{array}$ & Mortality $>50 \%$ \\
\hline Acute lupus pneumonitis (ALP) & $<10 \%$ & CXR: patchy infiltrates at bases & $70-90 \%$ \\
\hline \multicolumn{4}{|l|}{ Chronic } \\
\hline Chronic ILD & $3 \%$ & $\begin{array}{l}\text { CXR/CT: interstitial infiltrates, ground-glass } \\
\text { shadowing, honeycombing } \\
\text { PFT: } \downarrow \text { FVC, } \downarrow D_{\text {LCO }}\end{array}$ & $\begin{array}{c}\text { Variable, can be slowly } \\
\text { progressive }\end{array}$ \\
\hline Pulmonary hypertension & $5-14 \%$ & $\begin{array}{l}\text { Echocardiography: } \uparrow \text { right ventricular } \\
\text { pressures, PAP }>20 \mathrm{mmHg} \\
\text { PFT: stable FVC, } \downarrow D_{\text {LCO }}\end{array}$ & Up to $50 \%$ \\
\hline Shrinking lung syndrome & $<1 \%$ & $\begin{array}{l}\text { CXR: } \downarrow \text { lung volume, raised hemi diaphragm } \\
\text { PFT: } \downarrow \text { FVC, } \downarrow D_{\text {LCO }}\end{array}$ & Good \\
\hline
\end{tabular}

CXR: chest radiography; BAL: bronchoalveolar lavage; CTPA: computed tomography pulmonary angiogram; PFT: pulmonary function test.

up to $40 \%$ have associated lung involvement, and up to $80 \%$ of paediatric patients with JSLE will experience chest complications at some point in the disease course [3]. Pleuritic chest pain is the common presenting complaint. The most common histological pattern is nonspecific interstitial pneumonia, but any pattern can be seen [14].

The pulmonary complications in JSLE can be acute or chronic (table 1).

\section{Acute pulmonary complications in JSLE}

The pleura is the most commonly affected part of the respiratory system in JSLE. Pleuritis occurs in $80 \%$ of children with JSLE [3]. In fact, serositis, which is pleuritis with effusion, is one of the diagnostic criteria for JSLE [15]. The pleural effusion can be bilateral or unilateral. Chest pain is the most common presenting respiratory symptom, whereas complaints about shortness of breath are rare [16]. A pleural rub may be heard on auscultation. Many children remain asymptomatic [17]. The prognosis for isolated pleuritis is generally good.

Serositis is usually diagnosed with plain chest radiography, which demonstrates pleural effusion. Examples of pleural effusion and pericardial effusions on chest imaging in a child with JSLE are shown in figure 1.

The C-reactive protein (CRP) is often high in contrast to the normal or minimally high levels observed in patients with other manifestations of active JSLE $[18,19]$.
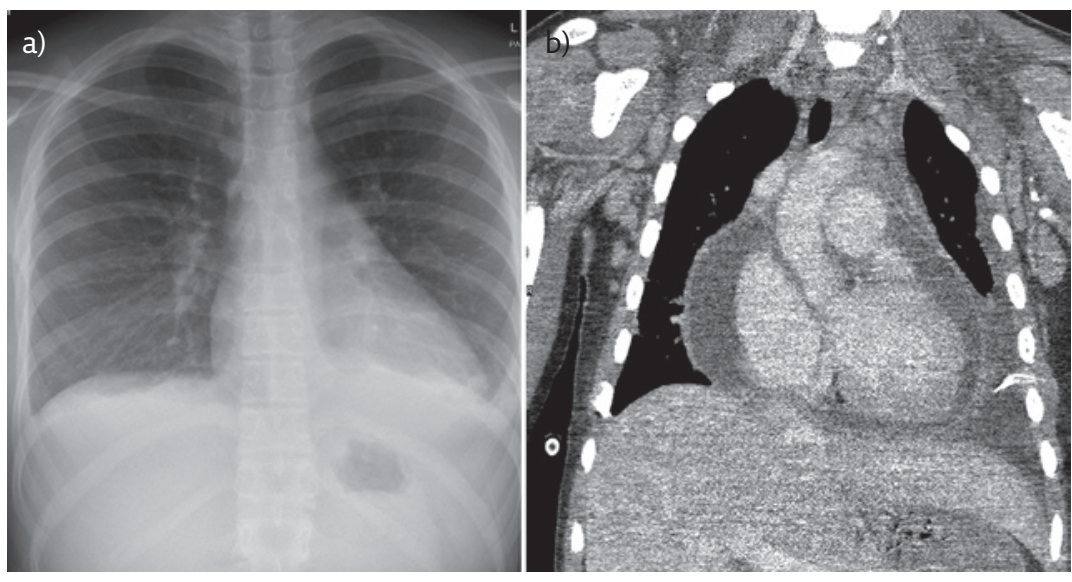

Figure 1 a) Chest radiograph of child with JSLE: the PA radiograph demonstrates normal lung volumes with bilateral pleural effusions, left to greater extent than right with subjacent compressive atelectasis. b) Chest computed tomography of another child with JSLE: coronal reconstruction from contrast-enhanced CT demonstrates pericardial and pleural effusion a feature of serositis. The pulmonary trunk is dilated, a feature seen in pulmonary hypertension. There is also evidence of left axillary lymphadenopathy. 
Pleural effusions may also occur due to nonrespiratory complications of JSLE, such as heart failure, or hypoalbuminaemia due to renal disease. Pulmonary infarcts or infection can cause haemorrhagic effusions associated with hypoxaemia [20].

The most common and severe acute lifethreatening pulmonary complication of JSLE is acute lupus pneumonitis (ALP). It is rare, with a reported incidence of $1-4 \%$, but with a mortality rate of $70-90 \%$ [14]. Children usually present with fever and respiratory distress. Inflammatory infiltrates in the interstitium and the alveolar wall cause diffuse alveolar damage, which is the hallmark of ALP [20]. Interestingly, ALP can be the initial presenting complaint of JSLE in half of the cases who develop this complication [14].

Although rare, alveolar haemorrhage $(\mathrm{AH})$ is also an emergency in JSLE [21]. The child can present with shortness of breath, pyrexia, haemoptysis and a rapid decline in respiratory function. Any child with JSLE with respiratory symptoms and a drop in haemoglobin should be investigated for an $\mathrm{AH}$. These children have high anti-dsDNA titres, and $90 \%$ of them have associated renal disease [3] Other considerations for patients with JSLE and haemoptysis are infection and pulmonary embolism especially if there is underlying antiphospholipid antibody syndrome.

Thromboembolic disease is the most common complication of antiphospholipid antibody syndrome, and these children can experience dyspnoea, pleuritic chest pain and haemoptysis [22]. Risk factors include smoking, oestrogencontaining contraceptive pills and proteinuria from nephrotic disease.

In JSLE, subclinical lung disease is present in $60-70 \%$ of patients who undergo pulmonary function tests, for example restrictive lung disease or reduced $D_{\text {LCO }}[23,24]$.
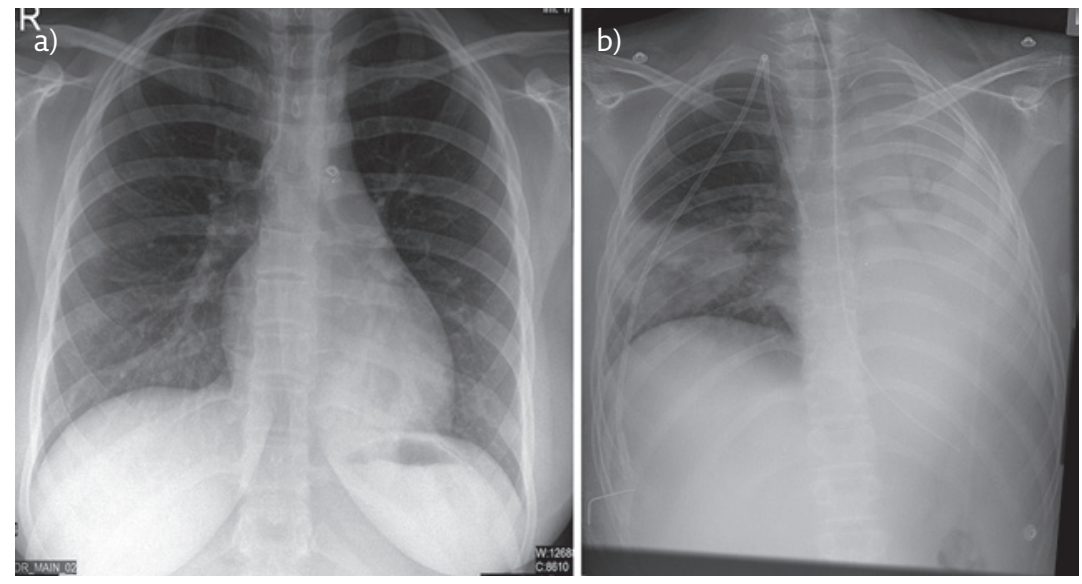

Figure 2 a) Chest radiograph of child with MCTD. This PA chest radiograph demonstrates retrocardiac left lower lobe consolidation; note increased retrocardiac opacity, indistinct bronchovascular markings and loss of cardiophrenic silhouette. Patent ductus arteriosus occluder device is in situ. b) Chest radiograph of a child with JDM with aspiration pneumonia. There is near total white out of the left hemithorax as a result of dense collapse consolidation of the left lung. There is wedge shaped consolidation in the right mid to lower zone.
In JSLE, patients are prone to infections with common viral and bacterial organisms, as well as opportunistic fungal infections (including Pneumocystis jirovecii), and rarer viral agents such as cytomegalovirus. Several factors contribute to this increased risk including reduced pulmonary macrophage and phagocytic function, abnormal complement cell-mediated immunity, lymphopenia, immunomodulator medication, and even a lower airway clearance from muscle weakness. The lung interstitium is uncommonly affected in JSLE during the acute presentations, except in these pulmonary infections [20].

Any of these pulmonary complications in JSLE may occur independently or simultaneously, for example ALP and AH coexist with pleuritis [25]. They all carry a high mortality rate, with over half of the affected patients dying as a result [26].

\section{Chronic pulmonary complications of JSLE}

Chronic ILD is rare in JSLE, but it may occur after ALP [27]. These children present with a gradual onset chronic dry cough, pleuritic chest pain and exertional breathlessness. Anti-smooth muscle and anti-ribonucleoprotein antibodies are believed to increase the risk of ILD [25].

Chronic pulmonary hypertension in JSLE manifests as shortness of breath, with associated desaturation rapidly progressing to right-sided cardiac failure. Causes include chronic hypoxia in ILD, pulmonary vasculitis, chronic thromboembolism, and valvular cardiac involvement. Raynaud's phenomenon and the presence of antiphospholipid antibodies are risk factors for pulmonary hypertension in JSLE [28].

Shrinking lung syndrome in JSLE results in reduced lung volumes, with raised hemidiaphragms and basal atelectasis seen on chest radiography [29]. A proposed explanation for this is the progressive diaphragmatic and intercostal muscle weakness.

\section{Juvenile dermatomyositis}

JDM or idiopathic inflammatory myopathy (IIM) is the most common inflammatory myopathy in children. It has an incidence of 0.1 per 100000 children [30] with a peak incidence at 5-14 years old [31]. It is a multisystem disease that can present with proximal muscle weakness, skin rashes including Gottron papules and heliotrope rash, and raised skeletal muscle enzymes [32].

Primarily, it causes neuromuscular weakness which leads to pulmonary complications through hypoventilation and aspiration pneumonia (figure $2 b$ ); sequelae of dysphagia and gastro-oesophageal reflux [33]. All patients with JDM should be assessed for dyspnoea, dysphagia and dysphonia at every clinical review. 
As well as the complications related to weakness and/or dyscoordination, JDM-related ILD occurs in $\sim 10-20 \%$ of children. The most commonly described pathological findings in JDM-related ILD is nonspecific interstitial pneumonia and cryptogenic organising pneumonia [14]. While some of these children may be asymptomatic, others may experience a dry cough and worsening shortness of breath. JDM-related ILD bears a poor prognosis with $30 \%$ of affected patients dying within 5 years of diagnosis [3]. High serum levels of Krebs von den Lungen (KL-6), anti-melanoma differentiation-associated gene 5 (MDA5) antibodies, and interleukin (IL)-19 are associated with rapidly progressive ILD [34]. The presence of anti-Jo- 1 antibodies is also a strong risk factor for developing this complication [35].

\section{Juvenile systemic sclerosis}

JSSc is typically characterised by symmetrical fibrous thickening and hardening of the skin combined with fibrous changes in internal organs such as the oesophagus, heart, kidneys and lungs [36].

The estimated incidence of JSSc ranges from 0.27 to 2.9 cases per million children per year [37]. The mean age of onset is 8-11 years old [38].

Severe lung disease is one of the major causes of death in children with JSSc. ILD is commonly found in JSSc, with rates of $65-90 \%$ in children. Nonspecific interstitial pneumonia is the most common form of ILD in JSSc [14].

\section{Mixed connective tissue disease}

MCTD was first described in 1972 as a CTD characterised by the presence of a specific autoantibody, now called anti-U1 ribonucleoprotein (RNP) [39]. It is often described as an "overlap syndrome" as it is associated with anti-U1 RNP antibodies and certain clinical features of JSLE, JSSC and JDM [40]. It often takes several years to diagnosis as the clinical features develop over time.

MCTD is rare in paediatrics, constituting up to $0.5 \%$ of the rheumatology workload [41].

Pulmonary complications occur in $75 \%$ adult patients with MCTD, and the proportion of affected children are believed to be comparable [3]. The complications include pleural effusions, pulmonary fibrosis and pulmonary hypertension, and are associated with a significant increase in morbidity and mortality [28].

\section{Sjögren's syndrome}

Sjögren's syndrome is a chronic multisystem condition characterised by a triad of xerostomia (dry mouth), xeropthalmia (dry eyes) and arthritis.
It can be primary or secondary to conditions such as lupus [42].

The prevalence of ILD in Sjögren's syndrome varies from $9 \%$ to $75 \%$ of patients and is most commonly seen in secondary Sjögren's syndrome. Nonspecific interstitial pneumonia is the most common pattern of disease, but lymphocytic interstitial pneumonia is also seen [14].

\section{Investigations}

Pulmonary investigations in CTD have multiple roles including screening, diagnosis, and monitoring of disease progression and complications of treatment.

\section{Blood tests}

Acutely, raised inflammatory markers and white cell counts can indicate active inflammation, but also infection which is extremely important to exclude in these conditions.

A few specific autoantibodies (e.g. anti-RNP, anti-Jo-1) are associated with pulmonary disease in CTD, but their titres may not systematically correlate with disease activity [7].

\section{Pulmonary function testing}

PFT is useful for detecting subclinical disease, for assessing disease severity, and for long-term monitoring of respiratory complications. They include $\mathrm{FVC}, \mathrm{FEV}_{1}$ and $\mathrm{FEV}_{1} / \mathrm{FVC}$ ratio, maximal inspiratory and expiratory pressures, as well as $D_{\mathrm{LCO}}$ [43]. Abnormalities in PFTs may precede radiological changes. Regular outpatient lung function testing will detect pulmonary complications of CTD early, which will enable clinicians to promptly manage these conditions.

A restrictive spirometry (figure 3 ) is commonly seen in JSLE-associated ILD. An increasingly restrictive pattern may indicate its progression [44].

Children with pulmonary involvement in JDM may display restrictive or occasionally obstructive patterns; there is a restrictive pattern in the spirometry of $8 \%$ of patients with asymptomatic JDM [45].

In the clinic setting, $D_{\mathrm{LCO}}$ is a sensitive indicator of pulmonary involvement, and impairment in $D_{\mathrm{LCO}}$ can precede abnormal radiological findings. $D_{\mathrm{LcO}}$ is decreased in ILD and may be low in almost half of patients with CTD, but this is not specific for ILD as it can happen secondary to pulmonary hypertension, which may also occur in CTD.

A recent study showed that $20 \%$ of patients with JSLE who underwent screening had a significantly reduced $D_{\text {Lco }}$ [46]. Acutely, an increase in $D_{\text {Lco }}$ can suggest $\mathrm{AH}$, which is an acute complication of JSLE [47]. Shrinking lung syndrome in JSLE is characterised by a restrictive spirometry, and a normal or increased $D_{\text {LCO }}$ [29]. 


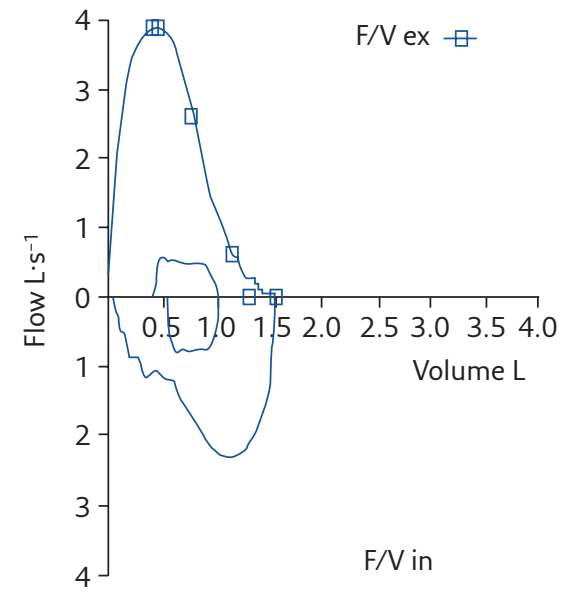

Spirometry
Substance
Dose
FEV 1 L
FVC L
VC max L
FEV $\%$ VC max \%
PEF
Lung volumes (He dilution)
RV-He L
FRC-He $\mathrm{L}$
TLC-He $\mathrm{L}$
Gas transfer
$D_{\mathrm{LCOs}} \mathrm{SB} \mathrm{mmol}^{-1} \mathrm{~min}^{-1} \cdot \mathrm{kPa}^{-1}$
$K_{\mathrm{CO}} \mathrm{mmol} \cdot \mathrm{min}^{-1} \cdot \mathrm{kPa}^{-1} \mathrm{~L}$
$V_{\mathrm{A}} \mathrm{L}$
$\mathrm{Hb}$ g per $100 \mathrm{~mL}$

$\begin{array}{llccc}\text { Pred } & \text { Pre } & \text { SR } & \text { \%Pred Post } \\ & & & & \\ 2.13 & 1.33 & -2.73 & 63 & \\ 2.49 & 1.54 & -3.00 & 62 & \\ 2.56 & 1.54 & -3.39 & 60 & \\ 84.56 & 86.38 & 0.33 & 102 & \\ 298.74 & 234.71 & -1.05 & 79 & \\ & & & & \\ \text { Pred } & \text { Pre } & \text { SR } & \text { \%Pred } & \text { Post } \\ 0.88 & 0.56 & -1.55 & 63 & \\ 1.61 & 1.21 & -1.40 & 75 & \\ 3.44 & 2.10 & -3.82 & 61 & \\ & & & & \\ \text { Pred } & \text { Pre } & \text { SR } & \text { \%Pred } & \text { Post } \\ 6.52 & 3.04 & -4.19 & 47 & \\ & 1.47 & & & \\ & 2.07 & & & \end{array}$

Figure 3 Pulmonary function testing in a patient with JSSC showing a restrictive pattern and reduced $D_{L C O}$. VC: vital capacity; PEF: peak expiratory flow; FRC: functional residual capacity; TLC: total lung capacity; $K_{\text {co: }}$ transfer coefficient of the lung for carbon monoxide; $V_{A}$ : alveolar volume; $\mathrm{Hb}$ : haemoglobin. For standardised residuals (SR): a value of $>1.64$ is the upper limit of normal, $<-1.64$ is below the lower limit of normal. Severity scale: mild $=-1.64$ to $-2.5 ;$ moderate $=$ -2.5 to -3.5 ; severe $=<-3.5$.

The frequency of screening and monitoring for pleuropulmonary complications in CTD remains controversial. Once lung disease is detected, spirometry with FVC, $\mathrm{FEV}_{1}$ and $\mathrm{FEV}_{1} / \mathrm{FVC}$ ratio should be undertaken every 3 months, and a full set of PFTs with $D_{\text {LCO }}$ can used longitudinally to monitor disease progression and remission every 6-12 months [7].

\section{Assessment of oxygenation}

Measuring oxygen saturation at rest and during exercise, as well as during sleep (through an overnight oximetry) helps to quantify the nature and the severity of the physiological impairment of gas exchange caused by the CTD-ILD [48]. The severity-of-illness score, described by FAN et al. [49], is determined at the initial assessment of children with ILDs and correlates with survival. A score of 1 to 5 is attributed as follows [49].

- Score 1: Asymptomatic

- Score 2: Symptomatic, normal room air oxygen saturation under all conditions

- Score 3: Symptomatic, normal resting room air saturation, but abnormal saturation $(<90 \%)$ with sleep or exercise

- Score 4: Symptomatic, abnormal resting room air saturation $(<90 \%)$

- Score 5: Symptomatic with pulmonary hypertension

Furthermore, the adequacy of oxygenation is being used as an outcome measure in current clinical trials, for example for the use of nintedanib in ILD [50].

\section{Imaging}

Plain film chest radiography may show consolidation or collapse, which is suggestive of infection, as exemplified in figure 2 , or pleural lines which indicate pleural effusions in cases of pleuritis [28].

In the clinic setting, chest radiography has a low sensitivity and specificity for diagnosing ILD in CTD. In JSLE, chest radiography changes include reticular interstitial infiltrates or honeycomb changes, but PFTs with $D_{\text {Lco }}$ are twice as sensitive at picking up abnormalities compared with chest radiography [3].

Typical chest radiograph changes, such as ground-glass opacities and reticular patterns, are only seen in $10-25 \%$ of children with JSSc-related lung disease [3].

\section{CT of the chest}

A chest $C T$ is more sensitive than a chest radiograph in the early detection of asymptomatic CTDrelated ILD. However, performing CT for detecting pulmonary complications in children with CTD is a controversial area [7]. In very young children, a chest CT may necessitate a general anaesthesia with inherent risk for the child. There are also concerns about the dose of radiation from a CT scan for a child, but evidence shows that the newer lowdose CT parameters can give valuable information to guide diagnosis of pulmonary complications in CTD [51].

An array of techniques can be employed to maximise the potential quality of $\mathrm{CT}$ imaging and hence improve interpretation of the results. Prone position CT scanning reduces posterior dependent atelectasis. In younger children, controlled ventilation CT optimises full inflation [51]. In older children, full-inflation inspiratory CT 
and spirometer-controlled full-inflation CT can be employed [7].

Most of the data on imaging in CTD comes from adult studies, and shows that a CT detects pulmonary changes in between $8 \%$ and $70 \%$ of adults with JSLE, where the most frequent abnormality detected was ILD [3]. These studies suggest that a CT is more sensitive at diagnosing ILD in asymptomatic patients when compared with PFT with $D_{\text {LCO. }}$.

Findings of ILD on CT initially show an inflammatory state, which includes groundglass opacities, reticular shadows and diffuse centrilobular nodularity. As ILD becomes more advanced, the CT changes denote a fibrotic state with honeycombing, bronchiectasis, and nonemphysematous cysts [52]. A typical chest CT of a teenager with JSSC is shown in figure 4. Staging of ILD on a CT scan can be classed as limited (involving $<20 \%$ of the lung parenchyma), borderline (20$25 \%$ lung involvement), or advanced (>25\% lung involvement) [53, 54].

In JDM, a case-control study showed that $37 \%$ of patients were found to have abnormalities on chest CT including ILD and airways disease, but also chest wall calcinosis. In that cohort, only $26 \%$ of children had restrictive patterns on $\mathrm{PFT}$, which shows that a CT may be more sensitive than PFT at detecting pulmonary complications [55].

CT imaging and Doppler ultrasounds are used to investigate for venous thromboembolic complications in children with JSLE acutely presenting with chest symptoms, especially in those having risk factors [22].

A chest CT may be a precursor investigation to guide a targeted BAL or a lung biopsy in CTD.

Not only is a chest CT diagnostic, it also aids the clinician in deciding on treatment options, guides the prognosis for the patient and helps in the monitoring of the response to treatment.

\section{Bronchoscopy}

In an acute setting, bronchoscopy allows the visualisation of the airway. An inflamed mucosa provides evidence of capillaritis, which occurs in $\mathrm{AH}$ in JSLE. BAL samples are usually bloodstained in $\mathrm{AH}$. Equally the presence of haemosiderin-laden macrophages even in non-bloodstained BAL shows that there has been recent bleeding [20].

Thick secretions on bronchoscopy may provide evidence of pulmonary infections. The BAL aids in the identification of the organism. The culture and sensitivity results help guide the antimicrobial regime. The BAL cytology helps to delineate inflammatory cells and may hence help to exclude infectious processes, and subsequently point to a diagnosis of ILD. A lymphocytic or eosinophilic predominance in the BAL cell count can indicate drug-induced organising pneumonia [7].

The lipid-laden macrophage index in the BAL sampling can complement the workup for other


Figure 4 Chest CT of a teenager with JSSc. a) Axial and b) coronal CT reconstructions: There is widespread intralobular septal thickening, predominating in the subpleural region. The apical segment of the left lower lobe demonstrates honeycombing indicating fibrosis. The fine subpleural ground-glass opacities in the right lower lobe indicates the presence of interstitial inflammation.

associated conditions which lead to recurrent chest infections in CTD, such as aspiration and gastrooesophageal reflux [56].

\section{Thoracentesis}

Needle aspiration of pleural effusions can be a useful diagnostic test. Pleural aspirates are exudative in pleuritis and empyema.

\section{Lung biopsy}

A lung biopsy may be indicated where the diagnosis is unclear, especially in the rarer childhood CTDs or in the presence of atypical features like hilar or mediastinal lymphadenopathy.

In JDM, due to the disease being so rare in children, a lung biopsy may still be needed to confirm the diagnosis of ILD, in addition to the highresolution CT [28]. 50\% of lung biopsies provide useful information to make a diagnosis of ILD in CTD. Typical ILD histopathology findings include desquamative interstitial pneumonia, nonspecific interstitial pneumonia and lymphocytic interstitial pneumonia [7].

A protocol for optimal lung biopsy outcome is detailed by the Children's Interstitial Lung Disease network [57]. The preferred approach is the use of a video-assisted thoracoscopic surgery, to obtain lung samples from various sites (a minimum of two) with the avoidance of the lingula and the tip of right middle lobe [52].

If a lung biopsy is needed, it is advisable to obtain it early in the course of pulmonary disease with the least amount of steroid use.

\section{Echocardiography}

It is recommended that all patients with JSLE undergo baseline echocardiography for early recognition of cardiac involvement, which can 
include pericarditis (commonest), myocarditis and non-infective endocarditis, and very rarely even pancarditis [58].

Pulmonary arterial hypertension (PAH) can be uncommonly associated with CTD [59, 60]. The clinical presentation of $\mathrm{PAH}$ can be nonspecific but can present with respiratory symptoms for e.g. tachypnoea, exercise intolerance, chest pain, syncope and progressive or refractory hypoxia [61]. Deterioration in CTD is usually rapid when associated with PAH and the presence of PAH in CTD attributes the highest score in severity-of-illness score [49].

If $\mathrm{PAH}$ is suspected, an echocardiography is performed. PAH is defined as a pressure $>20 \mathrm{mmHg}$ [59] and may complicate CTD. It can be suggested by an echocardiogram showing elevated right ventricular (RV) systolic pressures. The most precise echocardiographic method for measuring RV (and hence pulmonary artery) systolic pressure is to establish the velocity of the tricuspid regurgitant (TR) jet. The position of the interventricular septum at end systole is also a useful adjunct and offers a rough estimate of RV systolic pressure, but accurate quantification of pulmonary artery pressures is achieved only if a TR jet is measured [62].

\section{Gastrointestinal assessment}

Patients with CTD may suffer from gastrooesophageal reflux disease and aspiration, comorbidities which can further compromise lung health. Investigations which may help in the diagnosis include a pH study, an impedance study, an upper gastrointestinal contrast study or a videofluoroscopy [63]

\section{Treatment}

\section{General strategies}

The treatment goals in children with CTD include controlling the underlying inflammatory condition and the prevention and/or treatment of pulmonary complications.

A multidisciplinary team approach including respiratory paediatricians, paediatric rheumatologists, radiologists and pathologists is necessary in children with CTD for optimal outcomes.

Supportive care is essential. This includes adequate nutritional support, avoidance of secondhand smoke and e-cigarette vapour exposure and other irritants, the use of prophylactic antibiotics, prevention of infection through vaccination (e.g. the yearly influenza vaccine) and making sure that children have mounted a good response to their routine childhood vaccinations.

Supervised rehabilitation programmes and the provision of family support are important for a holistic approach to the child's care. In cases of severe ILD leading to inadequacy of oxygenation at rest and/or during sleep, long-term oxygen therapy is considered [64].

Due to the heterogeneity of CTD and some unanswered questions regarding the underlying aetiologies and pathogenesis, the evidence-based pharmacological treatments for the pulmonary manifestations in each condition varies.

\section{JSLE treatment grouped by complications}

Infection

As infection is the leading cause of death in patients with JSLE [65], all pulmonary changes should be considered infectious until proven otherwise. Therefore, a thorough and aggressive approach to investigation is required including bronchoscopy and lung biopsy. If a proven pathogen is isolated, this should be managed appropriately with the correct antibiotic choice. In some instances, an empirical antibiotic regime may be used.

\section{Pleuritis}

Mild cases can be managed with oral analgesia and oral steroids. In moderate to severe cases, patients usually require oxygen, analgesia and intravenous methylprednisolone for 3 days, followed by a short course of oral steroids. Although rare, refractory pleuritis and pleural effusions in JSLE have been treated with talc poudrage [66] or tetracycline pleurodesis [67].

\section{Acute pneumonitis}

This generally has a good response to steroids. However, if non-responsive to steroids and if chronic inflammation continues and shrinking lung syndrome develops, another immunosuppressant may be required, depending on the CTD and previous treatments if any.

\section{Acute pulmonary haemorrhage}

This is a life-threatening event that often requires ventilation and high-dose intravenous methylprednisolone. This is a sign of poor disease control; therefore, escalation of treatment is required. Cyclophosphamide has shown significant improvement in symptoms and lung function [16]. Rituximab, a monoclonal antibody which works by removing B-cells, may be helpful in reducing the frequency of recurrences of acute pulmonary haemorrhage in JSLE $[68,69]$.

\section{ILD}

There is no consensus on the pharmacological treatment of ILD. This may be due to the rarity of the disease, the difficulty in obtaining histological confirmation of ILD, and the various types of ILD. 
ILD presents with varying degrees of histology from inflammation to fibrosis and a wide spectrum of clinical manifestations from minimal symptoms to respiratory failure [14]. This makes it even more challenging for clinicians to make decisions regarding treatment options, observational strategies, optimal timings for interventions and the appropriateness of pharmacological agents for treatment [14].

As inflammation plays an important role in many forms of ILD, steroids are used in ILD [14]. There is a lack of evidence on the steroid regime that should be used in children; however, intravenous methylprednisolone is associated with fewer sideeffects [52]. Although not specific for paediatric CTDassociated ILD, a European protocol does provide guidance on treatment approaches in children. Steroid-sparing agents such as hydroxychloroquine, azathioprine, methotrexate, cyclophosphamide, mycophenolate mofetil (MMF) and biologics such as infliximab and rituximab have been used in ILD with varying success, but again with no clinical trials showing their efficacy in children [14, 70].

A paediatric clinical trial is currently being conducted on nintedanib [50], an intracellular inhibitor of tyrosine kinases, which has already proven to inhibit processes involved in the progression of lung fibrosis in adults [71].

Those children with advanced ILD may require long-term oxygen therapy. Lung transplantation is an option for those children who progress to endstage lung disease.

The development of pulmonary hypertension in ILD is associated with a poor prognosis and poor survival. Treatment consists of immunosuppressive drugs, mainly steroids and cyclophosphamide, as well as a pulmonary vasodilator, such as bosentan, sildenafil and epoprostenol, and oxygen therapy [72, 73]. Early intervention is recommended [49].

\section{Treatment of other CTDs and their pleuropulmonary complications}

\section{JDM}

In 2012, a European initiative called Single Hub and Access point for paediatric Rheumatology in Europe (SHARE) reached a consensus on the management of JDM [74]. At the time of diagnosis or disease flare-up SHARE recommended intravenous methylprednisolone followed by oral steroids. This is in combination with a steroid-sparing agent. Methotrexate is the preferred choice and works well in maintaining remission at a dose of $\sim 15 \mathrm{mg} \cdot \mathrm{m}^{-2}$ once weekly as a subcutaneous injection.

Cyclophosphamide is recommended in severe JDM-associated ILD. If JDM appears refractory to treatment, there are multiple other treatment options including biologics like rituximab, changing to anti-tumour necrosis factor (TNF) (infliximab/ adalimumab) or using combination therapy with high dose methotrexate, ciclosporin $A$ and intravenous immunoglobulins [74].

\section{Self-evaluation questions}

1. Which of the following features on a chest $\mathrm{CT}$ is not typically found in CTD-ILD?
a) Ground-glass opacity
b) Reticular and reticulo-nodular pattern
c) Traction bronchiectasis
d) Atelectasis

2. Which of the following in a BAL would suggest alveolar haemorrhage as a complication of JSLE?
a) Elevated macrophage-laden lipid index
b) Presence of haemosiderin-laden macrophages
c) Cell cytology showing eosinophilia
d) Ziehl-Neelsen stain positive

3. Which of the following statements is incorrect regarding rituximab?

a) Rituximab is a monoclonal antibody that works by removing B-cells

b) Use of rituximab can lead to ILD

c) Live vaccines including influenza vaccine can be administered to patients receiving rituximab

d) Undertaking a Mantoux test is mandatory prior to starting rituximab

4. A child with ILD is seen in clinic. She has the skin features shown in figure 5 . What is the most likely underlying diagnosis?
a) JSLE
b) MCTD
c) JDM
d) JSSC

JSSC

The 2009 European League against Rheumatism (EULAR) recommendations for the treatment of JSSc recommends that cyclophosphamide should be considered for treatment of JSSc-related ILD, in particular for patients with progressive disease. This recommendation is based on two high-quality randomised controlled trials [75]. There are no published studies in children to date; however, in clinical practice cyclophosphamide is used.

MMF is an alternative treatment choice. It may be better tolerated and is associated with less toxicity than cyclophosphamide [76].
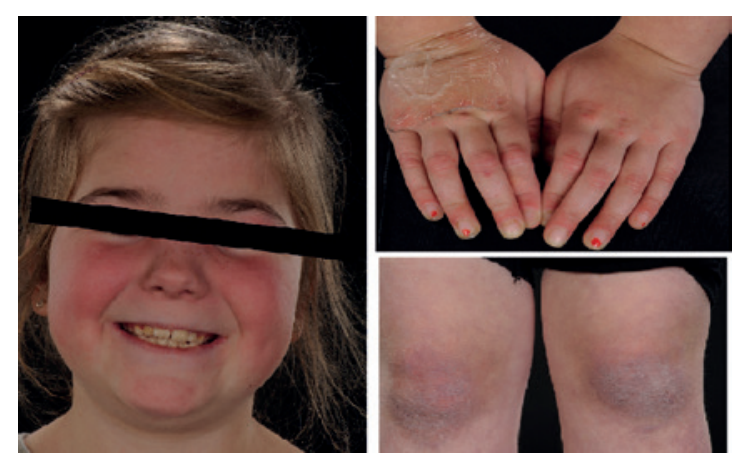

Figure 5 Skin rash in a child with ILD. 


\section{MCTD}

There is no specific treatment for MCTD. Those children with severe myositis and systemic involvement such as lung disease require high-dose steroids and cytotoxic drugs such as cyclophosphamide. Other drugs such as methotrexate, azathioprine, MMF and biological agents such as infliximab have been used [77]. Rituximab has also shown promising results [78].

\section{Sjögren's syndrome}

There is no clear consensus on treatment of ILD in Sjögren's syndrome. Steroids are often used. There is some evidence to suggest that azathioprine with and without steroids may be effective in treatment [79].

\section{Iatrogenic complications of CTD treatment}

The medications used in CTD are summarised in table 2.

Medications used to treat CTD may have undesirable pulmonary side-effects. It is important for clinicians to be aware of this as ongoing use worsens the pulmonary complications. It may be hard to differentiate between natural progression of CTD-ILD, for which the medication has been started in the first place, and iatrogenic complications.

Methotrexate can lead to pulmonary toxicity and this has been reported mostly in adults. It may lead to hypersensitivity pneumonitis, which has an incidence of 1-2\% [3], and patients experience shortness of breath, a dry cough and pyrexia. Interstitial or alveolar infiltrates may be seen on the chest radiograph. In addition, other complications such as reactive airways disease, pulmonary fibrosis, organising pneumonia and acute lung injury may be caused by methotrexate [20].

Biological DMARDs are a very effective treatment strategy for CTD, but can also cause ILD [80]. It is therefore crucial that any pre-existing ILD has been confirmed/excluded before commencing treatment with these agents.

Studies show that rituximab is associated with progression to ILD in adults. These patients often experience shortness of breath, pyrexia and tachypnoea with low oxygen saturation. The diagnosis is made by typical findings on a chest $C T$, along with a restrictive spirometry and decreased $D_{\mathrm{LCO}}$ [81]

Other immunosuppressant drugs used in CTD can have pulmonary complications which are summarised in table 3 [3, 81, 82]. However, these are uncommon in children. In such cases, the offending drug must be stopped and the child may need high-dose corticosteroids.

The use of immunosuppressant medication can favour opportunistic infections, which predispose

Table 2 Medications used in CTD

\begin{tabular}{ll}
\hline Medication & Mechanism of action \\
\hline $\begin{array}{l}\text { Corticosteroids } \\
\text { Prednisolone }\end{array}$ & Anti-inflammatory \\
Methylprednisolone & Anti-inflammatory \\
DMARDs & \\
Methotrexate & Dihydrofolate reductase inhibitor \\
Hydroxchloroquine & Lysosomal membrane stablisation, reduces IL-1 and TNF synthesis \\
Mycophenolate mofetil & Restricts T- and B-cell proliferation, acts on purine synthesising enzyme \\
Azathioprine & Metabolised to 6-mercaptopurine \\
Biologic therapy & \\
Etanercept & Soluble TNF- $\alpha$ receptor \\
Adalimumab & Monoclonal antibody to TNF- $\alpha$ \\
Infliximab & Monoclonal antibody to TNF- $\alpha$ \\
Canakinumab & Monoclonal antibody to IL-1 \\
Rituximab & Monoclonal antibody to CD20 \\
Abatacept & CTLA-4 fusion protein \\
Cytotoxic agent & Acts on all phases of cell cycle; acts on both T- and B-cells \\
Cyclophosphamide &
\end{tabular}


Table 3 Patterns of pulmonary injury caused by CTD medication

\begin{tabular}{ll}
\hline $\begin{array}{l}\text { Pattern of iatrogenic pulmonary } \\
\text { complication }\end{array}$ & Offending drug \\
\hline Pulmonary toxicity & Methotrexate \\
Interstitial pneumonitis & $\begin{array}{l}\text { Cyclophosphamide } \\
\text { Azathioprine } \\
\text { Cytokine modulators (etanercept, infliximab, rituximab) }\end{array}$ \\
Organising pneumonia & Methotrexate \\
Diffuse alveolar damage & Azathioprine \\
\hline
\end{tabular}

these children to lower respiratory tract infections. Latent tuberculosis (TB) may be activated. Investigations to rule out latent TB are undertaken before starting any biological therapy [65]. In addition to opportunistic infections, infections with common pathogens can present atypically or could be difficult to treat in these individuals and this should be borne in mind when managing these children.

\section{Conclusion}

Clinical presentations of autoimmune CTD are variable and can be associated with many pulmonary complications, as discussed in this review.
Acute complications must be managed promptly, and clinicians need to be aware of the propensity of these children to infection. Outpatient reviews undertaken jointly by respiratory and rheumatology paediatricians enable the early detection of pulmonary complications in asymptomatic patients. Children are offered extensive lung function testing and are referred for imaging appropriately.

Treatment strategies include the use of immunosuppressive medication, aiming to halt the progression of pulmonary disease. This model of multispecialty combined reviews aims to improve the prognosis for patients with CTD.

\section{Key points}

- Pleuropulmonary complications of CTDs are rare in the paediatric age group, but they are associated with high rates of morbidity and mortality.

- Many children initially may not experience respiratory symptoms, and unless clinicians are vigilant with a high degree of clinical suspicion, these complications may go undetected.

- Complications can be acute, potentially life-threatening (e.g. lower respiratory tract infection or alveolar haemorrhage), or chronic (e.g ILD and pulmonary hypertension) with significant morbidity.

- Pulmonary complications should be monitored by assessment of clinical symptoms, performing pulmonary function testing and through the use of various imaging modalities on a regular basis.

- Treatment includes steroids and other immunomodulators, including biological agents. To achieve the best possible outcome, affected children should be managed jointly by respiratory and rheumatology paediatricians.

Affiliations

Manisha Ramphul' ${ }^{1}$, Kathy Gallagher ${ }^{1}$, Kishore Warrier ${ }^{1}$, Sumit Jagani ${ }^{2}$, Jayesh Mahendra Bhatt ${ }^{1}$

'Dept of Paediatric Respiratory Medicine, Nottingham Children's Hospital, Nottingham University Hospitals, Nottingham, UK. 'Dept of Radiology, Nottingham University Hospitals, Nottingham, UK. 


\section{Conflict of interest}

None declared.

\section{Suggested answers}

1. d.

2. b.

3. c.

4. C.

\section{References}

1. Navallas M, Inarejos Clemente EJ, Iglesias E, et al. Connective tissue disorders in childhood: are they all the same? Radiographics 2019; 39: 229-250.

2. Femia A, Vleugels RA. Pediatric autoimmune connective tissue diseases: an update on disease characteristics, associations, and management. Curr Dermatol Rep 2013; 2: 216-229.

3. Richardson AE, Warrier K, Vyas H. Respiratory complications of the rheumatological diseases in childhood. Arch Dis Child 2016; 101: 752-758

4. Bahmer T, Romagnoli M, Girelli F, et al. The use of autoantibody testing in the evaluation of interstitial lung disease (ILD) - A practical approach for the pulmonologist. Respir Med 2016; 113: 80-92.

5. Shaw M, Collins BF, Ho LA, et al. Rheumatoid arthritisassociated lung disease. Eur Respir Rev 2015; 24: 1-16.

6. Kolahian S, Fernandez IE, Eickelberg O, et al. Immune mechanisms in pulmonary fibrosis. Am J Respir Cell Mol Biol 2016; 55: 309-322.

7. Cidon M, Bansal M, Hartl D. Pulmonary manifestations of rheumatologic diseases. Curr Opin Pediatr 2017; 29: 311-319.

8. Wynn TA. Integrating mechanisms of pulmonary fibrosis. J Exp Med 2011; 208: 1339-1350.

9. Vincze K, Kovats Z, Cseh A, et al. Peripheral CD4+ cell prevalence and pleuropulmonary manifestations in systemic lupus erythematosus patients. Respir Med 2014; 108: 766-774.

10. Kiboneka A. Paediatric systemic lupus erythematosus. An overview and current management. EC Paediatrics 2019; 8 : $171-178$.

11. O'Leary D, O'Connor C, Nertney L, et al. Juvenile systemic lupus erythematosus presenting as pancarditis. Pediatr Rheumatol Online J 2019; 17: 71

12. Kamphuis S, Silverman ED. Prevalence and burden of pediatric-onset systemic lupus erythematosus. Nat Rev Rheumatol 2010; 6: 538-546

13. Quinlan C, Santiago M, Smith L, et al. The effects of bethanechol on patients with tracheomalacia. Am J Respir Crit Care Med 2016; 193: A3843.

14. Vece TJ, Fan LL. Interstitial lung disease in children older than 2 years. Pediatr Allergy Immunol Pulmonol 2010; 23 : 33-41.

15. Hochberg MC. Updating the American college of rheumatology revised criteria for the classification of systemic lupus erythematosus. Arthritis Rheum 1997; 40: 1725-1725.

16. Beresford MW, Cleary AG, Sills JA, et al. Cardio-pulmonary involvement in juvenile systemic lupus erythematosus. Lupus 2005; 14: 152-158.

17. Çiftçi $E$, Yalçinkaya $F$, Ince $E$, et al. Pulmonary involvement in childhood-onset systemic lupus erythematosus: a report of five cases. Rheumatology 2004; 43: 587-591.

18. Firooz N, Albert D, Wallace D, et al. High-sensitivity C-reactive protein and erythrocyte sedimentation rate in systemic lupus erythematosus. Lupus 2011; 20: 588-597.

19. Ueki K, Ikeuchi H, Ota F, et al. Extremely high levels of $\mathrm{C}$-reactive protein in patients with acute lupus serositis. Mod Rheumatol 2002; 12: 267-270.

20. Rabinovich CE. Pulmonary Complications of Childhood Rheumatic Disease. Paediatr Respir Rev 2012; 13: 29-36.

21. Zamora MR, Warner ML, Tuder R, et al. Diffuse alveolar hemorrhage and systemic lupus erythematosus: clinical presentation, histology, survival, and outcome. Medicine (Baltimore) 1997; 76: 192-202.

22. Myones B, McCurdy D. The antiphospholipid syndrome: immunologic and clinical aspects. Clinical spectrum and treatment. J Rheumatol Supp/ 2000; 58: 20-28.

23. Al-Abbad A-J, Cabral DA, Sanatani S, et al. Echocardiography and pulmonary function testing in childhood onset systemic lupus erythematosus. Lupus 2001; 10: 32-37.
24. Trapani S, Camiciottoli G, Ermini M, et al. Pulmonary involvement in juvenile systemic lupus erythematosus: a study on lung function in patients asymptomatic for respiratory disease. Lupus 1998; 7: 545-550.

25. Keane MP, Lynch JP. Pleuropulmonary manifestations of systemic lupus erythematosus. Thorax 2000; 55: 159-166.

26. Wang L-C, Yang Y-H, Lu M-Y, et al. Retrospective analysis of mortality and morbidity of pediatric systemic lupus erythematosus in the past two decades. J Microbiol Immunol Infect 2003; 36: 203-208.

27. Quadrelli S, Alvarez C, Arce S, et al. Pulmonary involvement of systemic lupus erythematosus: analysis of 90 necropsies. Lupus 2009; 18: 1053-1060.

28. Dell S, Schneider R. Pulmonary involvement in the systemic inflammatory diseases of childhood. In: Wilmott RW, Boat TF, Bush A, et al., eds. Kendig and Chernick's Disorders of the Respiratory Tract in Children. Philadelphia, Elsevier, 2012; pp. 822-847.

29. Karim MY, Miranda LC, Tench CM, et al. Presentation and prognosis of the shrinking lung syndrome in systemic lupus erythematosus. Semin Arthritis Rheum 2002; 31: 289-298.

30. Quartier P, Gherardi RK. Chapter 149 - Juvenile dermatomyositis. In: Dulac O, Lassonde M, Sarnat HB, eds. Handbook of Clinical Neurology. Amsterdam, Elsevier, 2013; pp. 1457-1463

31. Kobayashi N, Takezaki S, Kobayashi I, et al. Clinical and laboratory features of fatal rapidly progressive interstitial lung disease associated with juvenile dermatomyositis. Rheumatology (Oxford) 2015; 54: 784-791.

32. Martin N, Li CK, Wedderburn LR. Juvenile dermatomyositis: new insights and new treatment strategies. Ther Adv Musculoskelet Dis 2012; 4: 41-50.

33. Pouessel G, Deschildre A, Le Bourgeois M, et al. The lung is involved in juvenile dermatomyositis. Pediatr Pulmonol 2013; 48: 1016-1025.

34. Rider LG, Lindsley CB, Miller FW. Chapter 26 - Juvenile Dermatomyositis. In: Petty RE, Laxer RM, Lindsley CB, et al., eds. Textbook of Pediatric Rheumatology (Seventh Edition) Philadelphia, W.B. Saunders, 2016; pp. 351-383.

35. Schnabel A, Reuter M, Biederer J, et al. Interstitial lung disease in polymyositis and dermatomyositis: Clinical course and response to treatment. Semin Arthritis Rheum 2003; 32: 273-284.

36. Zulian F. Scleroderma in children. Best Pract Res Clin Rheumato 2017; 31: 576-595.

37. Royle JG, Lanyon PC, Grainge MJ, et al. The incidence, prevalence, and survival of systemic sclerosis in the UK Clinical Practice Research Datalink. Clin Rheumatol 2018; 37: 2103-2111.

38. Martini G, Foeldvari I, Russo R, et al. Systemic sclerosis in childhood: Clinical and immunologic features of 153 patients in an international database. Arthritis Rheum 2006; 54: 3971-3978.

39. Sharp GC, Irvin WS, Tan EM, et al. Mixed connective tissue disease-an apparently distinct rheumatic disease syndrome associated with a specific antibody to an extractable nuclear antigen (ENA). Am J Med 1972; 52: 148-159.

40. Berard RA, Laxer RM. Pediatric mixed connective tissue disease. Curr Rheumatol Rep 2016; 18: 28.

41. Bowyer S, Roettcher P. Pediatric rheumatology clinic populations in the United States: results of a 3 year survey. Pediatric Rheumatology Database Research Group. J Rheumatol 1996; 23: 1968-1974

42. Tucker L. Chapter 30 - Sjögren Syndrome. In: Petty RE, Laxer RM, Lindsley CB, Wedderburn LR, eds. Textbook of Pediatric Rheumatology (Seventh Edition). Philadelphia, W.B. Saunders, 2016; pp. 427-435. 
43. Alkady EAM, Helmy HAR, Mohamed-Hussein AAR. Assessment of cardiac and pulmonary function in children with juvenile idiopathic arthritis. Rheumatol Int 2012; 32: 39-46

44. Alamoudi OSB, Attar SM. Pulmonary manifestations in systemic lupus erythematosus: association with disease activity. Respirology 2015; 20: 474-480.

45. Mathiesen PR, Buchvald F, Nielsen KG, et al. Pulmonary function and autoantibodies in a long-term follow-up of juvenile dermatomyositis patients. Rheumatology (Oxford) 2014; 53: 644-649.

46. Lilleby V, Aaløkken M, Johansen B, et al. Pulmonary involvement in patients with childhood-onset systemic lupus erythematosus. Clin Exp Rheumatol 2006; 24: 203-208.

47. Solomon JJ, Fischer A. Connective tissue disease-associated interstitial lung disease: a focused review. J Intensive Care Med 2015; 30: 392-400.

48. Kuo CS, Young LR. Interstitial lung disease in children. Curr Opin Pediatr 2014; 26: 320-327.

49. Fan LL, Kozinetz CA. Factors influencing survival in children with chronic interstitial lung disease. Am J Respir Crit Care Med 1997; 156: 939-942.

50. ClinicalTrials.gov. A study to find out how nintedanib is taken up in the body and how well it is tolerated in children and adolescents with interstitial lung disease (ILD). NCT04093024. https://clinicaltrials.gov/ct2/ show/NCT04093024?term=fibrosing+\%2C+children\&cond= nintedanib\&draw=2\&rank=1 Date last accessed: 3 July 2020.

51. Semple T, Owens CM. The radiology of diffuse interstitial pulmonary disease in children: pearls, pitfalls and new kids on the block in 2015. Radiol Med 2016; 121: 352-361.

52. Bush A, Cunningham S, de Blic J, et al. European protocols for the diagnosis and initial treatment of interstitial lung disease in children. Thorax 2015; 70: 1078-1084.

53. Doyle TJ, Lee JS, Dellaripa PF, et al. A roadmap to promote clinical and translational research in rheumatoid arthritisassociated interstitial lung disease. Chest 2014; 145: 454-463.

54. Goh NSL, Desai SR, Veeraraghavan S, et al. Interstitial lung disease in systemic sclerosis: a simple staging system. Am Respir Crit Care Med 2008; 177: 1248-1254.

55. Sanner H, Aaløkken TM, Gran JT, et al. Pulmonary outcome in juvenile dermatomyositis: a case-control study. Ann Rheum Dis 2011; 70: 86-91.

56. Kazachkov MY, Muhlebach MS, Livasy CA, et al. Lipid-laden macrophage index and inflammation in bronchoalveolar lavage fluids in children. Eur RespirJ 2001; 18: 790-795.

57. Langston C, Patterson K, Dishop MK, et al. A protocol for the handling of tissue obtained by operative lung biopsy: recommendations of the chILD Pathology Co-Operative Group. Pediatr Dev Pathol 2006; 9: 173-180.

58. Watson L, Leone V, Pilkington C, et al. Disease activity, severity, and damage in the UK juvenile-onset systemic lupus erythematosus cohort. Arthritis Rheum 2012; 64: 2356-2365.

59. Rosenzweig EB, Abman SH, Adatia I, et al. Paediatric pulmonary arterial hypertension: updates on definition, classification, diagnostics and management. Eur Respir J 2019; 53: 1801916

60. del Cerro MJ, Abman S, Diaz G, et al. A consensus approach to the classification of pediatric pulmonary hypertensive vascular disease: report from the PVRI Pediatric Taskforce, Panama 2011. Pulm Circ 2011; 1: 286-298.

61. Avitabile CM, Vorhies EE, Ivy DD. Drug treatment of pulmonary hypertension in children. Paediatr Drugs 2020; 22: 123-147

62. Hansmann G, Apitz C, Abdul-Khaliq H, et al. Executive summary. Expert consensus statement on the diagnosis and treatment of paediatric pulmonary hypertension. The European Paediatric Pulmonary Vascular Disease Network, endorsed by ISHLT and DGPK. Heart 2016; 102: Suppl. 2 ii86-i100.
63. Vandenplas Y, Rudolph CD, Di Lorenzo C, et al. Pediatric Gastroesophageal Reflux Clinical Practice Guidelines: Joint Recommendations of the North American Society for Pediatric Gastroenterology, Hepatology, and Nutrition (NASPGHAN) and the European Society for Pediatric Gastroenterology, Hepatology, and Nutrition (ESPGHAN). J Pediatr Gastroenterol Nutr 2009; 49: 498-547

64. Clement A, Nathan N, Epaud R, et al. Interstitial lung diseases in children. Orphanet J Rare Dis 2010; 5: 22-22.

65. Faco MMM, Leone C, Campos LMA, et al. Risk factors associated with the death of patients hospitalized for juvenile systemic lupus erythematosus. Braz J Med Biol Res 2007; 40: 993-1002.

66. Kaine JL. Refractory massive pleural effusion in systemic lupus erythematosus treated with talc poudrage. Ann Rheum Dis 1985; 44: 61-64.

67. McKnight KM, Adair NE, Agudelo CA. Successful use of tetracycline pleurodesis to treat massive pleural effusion secondary to systemic lupus erythematosus. Arthritis Rheum 2010; 34: 1483-1484

68. Nellessen CM, Poge U, Brensing KA, et al. Diffuse alveolar haemorrhage in a systemic lupus erythematosus patient successfully treated with rituximab: a case report. Nephrol Dial Transplant 2007; 23: 385-386

69. Narshi CB, Haider S, Ford CM, et al. Rituximab as early therapy for pulmonary haemorrhage in systemic lupus erythematosus. Rheumatology (Oxford) 2010; 49: 392-394.

70. Mathai SC, Danoff SK. Management of interstitial lung disease associated with connective tissue disease. BMJ 2016; 352: h6819.

71. Rivera-Ortega P, Hayton C, Blaikley J, et al. Nintedanib in the management of idiopathic pulmonary fibrosis: clinical trial evidence and real-world experience. Ther Adv Respir Dis 2018; 12: 1753466618800618

72. Tselios K, Gladman D, Urowitz M. Systemic lupus erythematosus and pulmonary arterial hypertension: links, risks, and management strategies. OARRR 2016; 9: 1-9.

73. Mok MY, Tsang PL, Lam YM, et al. Bosentan use in systemic lupus erythematosus patients with pulmonary arterial hypertension. Lupus 2007; 16: 279-285.

74. Bellutti Enders F, Bader-Meunier B, Baildam E, et al. Consensus-based recommendations for the management of juvenile dermatomyositis. Ann Rheum Dis 2017; 76: 329-340.

75. Kowal-Bielecka O, Fransen J, Avouac J, et al. Update of EULAR recommendations for the treatment of systemic sclerosis. Ann Rheum Dis 2017; 76: 1327-1339.

76. Tashkin DP, Roth MD, Clements PJ, et al. Mycophenolate mofetil versus oral cyclophosphamide in scleroderma-related interstitial lung disease (SLS II): a randomised controlled, double-blind, parallel group trial. Lancet Respir Med 2016; 4: 708-719.

77. Deepak S, Warrier KC. Mixed connective tissue disease in children - case series. Rheumatol Orthop Med 2017; 2: doi: 10.15761/ROM.1000118.

78. Jovancevic B, Lindholm C, Pullerits R. Anti B-cell therapy against refractory thrombocytopenia in SLE and MCTD patients: long-term follow-up and review of the literature. Lupus 2013; 22: 664-674.

79. Deheinzelin D, Capelozzi VL, Kairalla RA, et al. Interstitial lung disease in primary Sjögren's syndrome. Clinicalpathological evaluation and response to treatment. Am J Respir Crit Care Med 1996; 154: 794-799.

80. Chen J, Chi S, Li F, et al. Biologics-induced interstitial lung diseases in rheumatic patients: facts and controversies. Expert Opin Biol Ther 2017; 17: 265-283.

81. Lioté $H$, Lioté $F$, Séroussi B, et al. Rituximab-induced lung disease: a systematic literature review. Eur Respir J 2010; 35: 681.

82. Roubille C, Haraoui B. Interstitial lung diseases induced or exacerbated by DMARDS and biologic agents in rheumatoid arthritis: A systematic literature review. Semin Arthritis Rheum 2014; 43: 613-626. 